\title{
A Pleasurable Persuasive Model for E-fitness System
}

\author{
Lizhen $\operatorname{Han}^{1,2}$ \\ ${ }^{1}$ State Key Lab of CAD\&CG \\ Zhejiang University \\ Hangzhou, China \\ ${ }^{2}$ College of Design \\ Shandong University of Arts \\ Jinan, China \\ hlz-1@126.com \\ Zhigeng Pan \\ DMI Research Center \\ Hangzhou Normal University \\ Hangzhou, China \\ zgpan@hznu.edu.cn
}

\author{
Mingmin Zhang \\ Computer and Engineering Department \\ Zhejiang University \\ Hangzhou, China \\ zhangmm95@zju.edu.cn
}

Feng Tian

School of Design, Engineering \& Computing

Bournemouth University

Bournemouth BH, UK

ftian@bournemouth.ac.uk

\begin{abstract}
The regular physical activity with enough amounts of intensity, duration and frequency plays a key role in our health and body shape. However, for sedentary individuals who have negative or even painful feeling on exercise, it is a challenge to maintain enough activities. We propose a pleasurable persuasive model (PPM) to tackle the challenge. PPM emphasizes the combination of psychological driven and physiological adaptation, encouraging health behavior change in an aesthetical pleasurable way. Coming with a complete set of design strategies including pleasure, guidance, motivation, and reminder, a prototype system is developed to experiment the effectiveness and feasibility of the approach. The real time biofeedback and aesthetical pleasure is integrated seamlessly into this system. Results from a three-week in-lab user study have demonstrated that, the system is able to encourage regular physical activities with enough amounts of intensity, duration and frequency in a pleasurable way, proving the effectiveness of PPM.
\end{abstract}

Keywords-HCI; persuasive model; aesthetical pleasure; real time biofeedback; health behavior change; exergame

\section{INTRODUCTION}

Our lives have become more efficient and convenient in the digital era compared to the traditional age. However, the digital era is likely to lead to a sedentary lifestyle. Many diseases, such as heart disease and obesity, both of which are harmful to a person's general health, are linked to such a lifestyle [1].

Regular performance plays a key role in human body shape and health condition. Doing regular physical activity with enough amounts of intensity, duration and frequency is a healthy lifestyle. According to the recommendation of ACSM (American College of Sports Medicine), healthy adults need "moderate-intensity aerobic physical activity for a minimum of 30 minutes on five days each week, or vigorous-intensity aerobic activity for a minimum of 20 minutes on three days each week, or the combinations of these two"[ 2$]$.
Unfortunately, most of the public fail to meet the minimum dose[3].

Intensive research has been done to help people change their sedentary behavior (e.g.[4] [5]). Game-related exercise, which often called exergame, is conducive to increase the amounts of physical activity. However, the amounts of exercise are not simply the more the better. On the other hand, given different body state and health condition, the intensity level of each person is different. So, in the pursuit of individual adaptability and adequate intensity of exercise, the persuasive fitness system design remains challenging.

We believe a good fitness system should suitable for personal physical condition, engage them to exercise regularly and scientifically with enough amounts of intensity, duration and frequency, furthermore, make them glad to persist on, so that they could happily change their life style from sedentary to active.

We propose a pleasurable persuasive model (PPM) to meet the design requirements of e-fitness system. PPM emphasizes the combination of psychological driven and physiological adaptation, to encourage personal, scientific and effective long term physical activity. The aesthetical pleasure is used as a major design strategy to combine the other three - guidance, encouragement, and reminder.

A prototype system is developed based on PPM to demonstrate our idea and to prove its feasibility. In such a system, the four design strategies are integrated into one indoor daily cycling system seamlessly, aiming to lead healthy behavior through a beautiful virtual world. Then we conduct a three-week in-lab user study to validate PPM.

This paper is organized as follows. In Section 2, we give a review on persuasive technology and systems as well as the usage of biofeedback in exergames. Then, we describe PPM in details in Section 3. The sample application based on PPM is illustrated in Section 4, followed by the user study in Section 
5. Finally, in Section 6 and 7, the discussion and general conclusion are drawn.

\section{RELATED WORK}

\section{A. Persuasive Technology}

Persuasive technologies are intentionally designed to change people's behavior, attitude or both[6]. This kind of change should be voluntary and, persuaded without using coercion or deception.

Many theories based persuasive technologies can be applied to health behavior change. The Goal-Setting Theory[7] and the Transtheoretical Model (TTM) of Behavior Change[8] are the two often used. By setting different types of health behavior goals, individuals tend to motivate and perform well[9]. TTM offers an integrative framework for understanding and intervening with human intentional behavior change through several stages, processes and levels. By using stage-matched interventions, the persuasive technology may get most effective outcome.

Fogg's behavior model (FBM)[10] is another persuasive theory, where behavior is a collaborative product of three factors: ability, motivation, and triggers. Fogg point out that these three factors must occur simultaneously, or the change of behavior will not happen. In FBM, the pleasure and pain is the first core motivator. Fogg believes that it is powerful and that the result of this motivator is immediate.

Taking advantages of these three theories and findings, especially form FBM, PPM makes some contributes to persuasive technologies. These contributions lie in: 1) proposing a feasible framework on how to design e-fitness system from a designer's point of view; 2) periodically using aesthetic pleasure to set the exercise goal; 3) combine aesthetic pleasure with other factors.

\section{B. Motivation of Exergame}

Exergame use a fun video game play way to motivate players to become more physically active [11] [12] [13]. Motivation is "an inquiry into the why of behavior"[14]. It is "an internal state or condition (sometimes described as a need, desire, or want) that serves to activate or energize behavior"'[15]. Motivation principle is an important aspect for effective persuasion. PPM concerns the way of incentive strategies being used in exergames, considering that a player's motivation is vital to a game's popularity as well as the effectiveness of exercise.

To encourage players to do more physical activity, some exergames track the player's action, emotion or bio-signal to control the game play (e.g. [16] [17]), or to auto-adapt the game level for fair game play experiences (e.g. [18][19][20]). Some set challenging goals to lure player spending more time (e.g. [21]). Others use competitions or award strategies to encourage more workout (e.g.[22]).

Most of the approaches above adopt compulsive incentive strategies to attract and retain players. This kind of motivation tend to spurred the players to try their best to win. However, high-intensity activities of an extended duration are not beneficial for a person's health as these activities may require the exerciser to maintain their heart rate at a desired level within the allocated time. In fact, few exergames follow the scientific guidelines of ACSM.

In this paper, by strictly following the guidelines of ACSM, we introduce aesthetical pleasure as a moderate while naturally encouragement to promote appropriate fitness.

\section{Particularly Related Works}

Consolvo, McDonald and Landay [23]propose eight design strategies for persuasive technologies to encourage physically active lifestyle. They use a mobile implementation called UbiFit Garden to validate the usefulness of abstract \& reflective, unobtrusive, public, aesthetic, positive, controllable, trending/historical and comprehensive. Their works mainly support for the individual's fundamental social needs through the intersection of technology. Aesthetic is used in its apparent level to enhance visual attraction, make context awareness, and give privacy protection. The main difference between our work and their work is that our model is designed differently. Our model includes the aesthetic as a major incentive strategy and integrates real-time biofeedback and virtual reality technology into our application.

Many commercial companies offer products that promote exercise by using the exercise equipment to control video games. A particular relevant one is the Expresso bike[24]. As a successful commercial system, Expresso bike combines multiple game play modes, such as updatable virtual environments, competitive virtual riding courses, and numerical presentation of heart rate, to encourage . Our prototype system takes the similar approach of virtual cycling, but mainly different in the association between physiological adaptation and aesthetic driven.

Overall, we can see that little consideration, neither in terms of persuasive technologies nor in terms of their applications, has been given to enable persons to cope with the painful feeling resulting from physical exertion. PPM tries to overcome this challenge by using a pleasurable persuading mechanism to avoid nervousness, anxiety, irritation, and despair.

\section{OUR METHOD- PleAsurable PERSUASIVE MODEL}

Regular exercise with enough amounts of intensity, duration and frequency is beneficial for health but difficult or even painful to maintain. PPM aims to act as a practical solution on encouraging healthy behavior in a pleasurable way. It comes with the following four design strategies:

\section{A. Pleasure}

Take pleasure as a powerful motivator to pursue target behavior as well as to transfer pain.

Pleasure is a nice feeling of satisfaction and enjoyment which makes parts or all of the human senses comfortable. It is opposite to pain and can be acted as a powerful motivator[10]. This kind of affective sensation is different from the exquisite joy of high emotion, but a relatively moderate delight which can be extended to a long time. 
Pleasurable persuasion could enhance users' self-efficacy by avoiding frustration from failure. Fitness system should give users sufficient and yet sustainable pleasurable experience to pursue enough amounts of regular performance. Pleasure from aesthetics is a good choice because beauty is commonly regarded as the source of pleasure.

The power of pleasure is the key strategy of PPM which integrates the following three into a whole.

\section{B. Guidance}

Give users ability to recognize their exercise intensity as well as inner body state, lead them to meet the target level in a pleasurable way.

This is the basic consideration to ensure the amount of exercise intensity. Cognition of intensity can increase user ability on doing exertion performance effectively and scientifically. Without such scientific guidance, users are hardly to learn about their body state accurately, especially for the internal state level of Responding Body[25]. Fitness system should give explicit context awareness of users' physiological state and provide explicit control for the adaptation of intensity.

The explicit tendency of the context-awareness should be given to lead users meeting the exercise goal. Progressive response or adaptive interface toward this kind of tendency is useful.

The power of pleasure should be better designed into the context-awareness and worked as a leading role towards the training goal.

\section{Encouragement}

Motivate users to maintain enough duration within the target zone happily.

A system intended to change health behavior should provide sufficient motivation to achieve exercise of appropriate duration. When designing a fitness system, the challenge is to engage users to sustain the optimal training level. Aesthetic pleasure can be taken into consideration. That is because the inherent essence of beauty, such as variation and novelty, intrinsically satisfies human needs.

\section{Reminder}

Call for long term regular exercise with enough frequency in a pleasurable way.

Long term regular exercise needs scientific management to obtain progressing health benefit through each time daily task performing. The history of performance data recording, accomplishment of exercise plan, or data analysis in a pleasurable way are some cases of practical using. This is an important factor that should be taken into consideration on designing long term fitness system for behavior change. Fitness management and attractive freshness of the system are quite useful in providing a trigger to remind the performance as in plan and recall for the next time.
All these four strategies of PPM are designed to encourage regular physical activity with enough doses in a pleasurable way. They also matched the three necessary conditions towards target behavior of Fogg's Behavior Model, as shown in Fig. 1.

\section{A PROTOTYPE SYSTEM IMPLEMENTATION}

A prototype system is developed based on PPM to demonstrate our idea and to prove its feasibility.

In the system, we chose cycling, a popular adaptive exercise mode, as our system exercise type for the ease of control of intensity. The selected bio-signal data is user's real time heart rate. heart rate data is the primary standard of the body state measurement. A set of heart rate monitor (Polar WearLink ${ }^{\circledR}+$ W.I.N.D. transmitter and Polar WindLink $\left.{ }^{\mathrm{TM}}\right)$ is used to capture and transfer the bio-data of the user. A virtual environment is displayed on a screen in front of a sensorembedded exer-bike (Fig. 2).

When cycling, the audiovisual effects of the system interface respond according to the user's physiological and physical data. As a result, the dynamical bio-signal data is visualized as a meaningful natural landscape. The visualization also adapts the speed of the virtual cyclist to the actual user performance. With a rational association between the different levels of exercise intensity and the progressive stylized display, an aesthetic pleasurable virtual world is generated. The more vivid and vigorous the appearance of the virtual environment, the healthier and livelier the user's body status becomes and, the more emotional pleasure the user experiences.

The prototype system also makes some systematic settings, including personal information input, customized scene's elements, and history recording, for a fitness management.

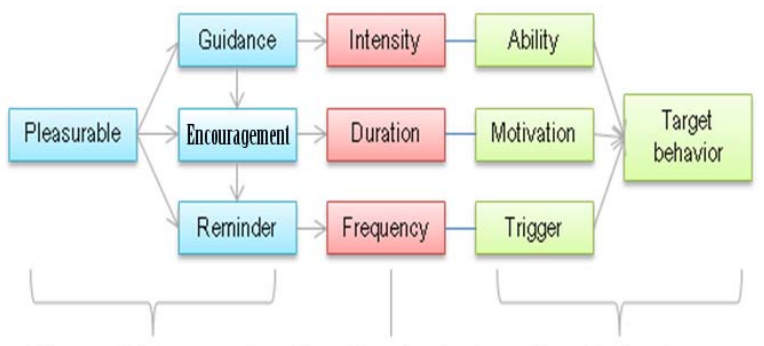

Pleasurable Persuasive Regular physical FoggBehavior

Fig. 1. The relationship between Pleasurable Persuasive Model, regular physical activity, and Fogg's Behavior Model.

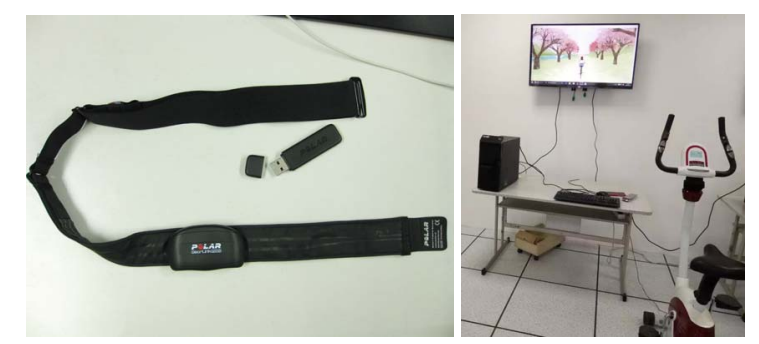

Fig. 2. Equipments of the prototype system. 
The description on how to apply PPM into fitness system design is given below in details.

\section{A. Pleasure}

We take aesthetical pleasure as the main power for behavior persuasion. Because beauty is a promise of happiness[26]. People seek for beauty instinctively. According to the views of visual thinking[27], the aesthetical pleasure is results from the reductions of tension or a balance of driven force. On the contrary, ugly often means full of tension and out of balance, which leads uncomfortable. In fact, beauty and ugly is relative.

A natural scene with trees, cloud, river, mountain and wild flower is designed as a sample to give awareness of the personalized body state. The balance and harmony of all these elements and, the tension of dynamic force from different condition, make up the aesthetic judgment (Fig. 3).

Besides the visual effects of the virtual scene, some audio effects are also set up to match the different heart rate zones. The audio effects aim to strengthen the awareness of exercise intensity and the affective experience.

\section{B. Guidance}

There are two missions in the system design at this part.

On how to give users ability to recognize their exercise intensity:

We couple the physiological measurement to the audiovisual effects of the system and set up a timely biofeedback. The captured raw heart rate data are transferred into the system and translated into five common used training zones[28] plus an invalid zone based on one's personal information. Using the metaphor of nature scene, we visualize the heart rate data into some blooming trees and the wildflower beside the way. The higher the heart rate is the more flowers are blooming. The heart rate data is also linked to the waves of river and the lightness of sky. The higher the heart rate is, the warmer and brighter the scene's sky light changes. By this way, direct physiological sensors are mapped intuitively to reflect the body state in the virtual world. The cycling speed and orientation are directly mapped into the virtual traveling. Sound effects including water flow and bird sing in different kinds are connected to the heart rate zones. Thus, the real-time generated presentation is full of context awareness, communicates the state of user's training. From adjust the pedal speed then influence the heart rate level, users could control the virtual environment appearance accordingly.

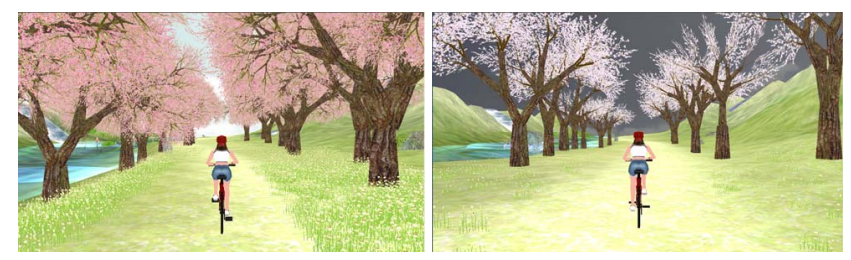

Fig. 3. A beautiful scene (left) gives more pleasurable sensation than a relativly ugly one gives.
On how to guide users to meet the target level in a pleasurable way:

Following the visual gestalt theory, we make progressive visualization of heart rate levels, and add aesthetic tendency in the interface presentation, as illustrated in Fig. 4. Gestalt psychology [29] implies that human mind tends to perceive external stimuli as whole rather than the sum of their parts, under the way of using some laws of grouping, such as law of similarity, law of closure, and law of continuity. In this case, a blooming tree is used to form the visual gestalt.

A popular aesthetical preference of the aesthetic tendency is being investigated in advance through online and offline investigation. In this investigation, more than 40 participants give their order of aesthetical pleasure to some random snapshots of our system. A well accepted tendency is being adopted (Fig. 5). We combine the most beautiful scene with the target heart rate zone, the invalid zone with the ugly one, and the rest so on in grade. The closer the heart rate matches the target zone, the more beautiful the environment looks like. Users can also set customized aesthetic tendency from the beginning of a user selection interface. Under the instinctive pursuit of beauty, users are engaged to build a beautiful virtual world instead of a less beautiful one.

\section{Encouragement}

To encourage users stay in the target training zone for a longer enough periods, we assign more tasks to beauty by adding awards of novelty to the system. That means, the system will reward users by making the virtual environment more aesthetically pleasing when they keep on staying at the target zone.

Our award system is also following the guidelines of ACSM. In the system, users have 5 minutes to warm up and get into the target heart rate zone. Within the 5 minutes, elements respond to the heart rate data normally. There is no award. When the user gets into the target zone and stays more than 3 minutes, one kind of wonderful flower will spread on the road-side as an award of level 1. The additional one minutes add, one random kind of flowers appear. One may never tell what kind of flowers will show. Five times later, a higher level of award will be given - a random kind of animal appearing in the front, as shown in Fig. 6. This kind of award is relatively more valuable than level 1 because it just takes place once at each time and lasts one minute. Then the award system backs to level 1 and repetitively gives random flowers until the user gets into level 2 twice to be awarded another random animal. We build an award library with lots of flowers and animals. The longer one maintain in the target zone,

a)
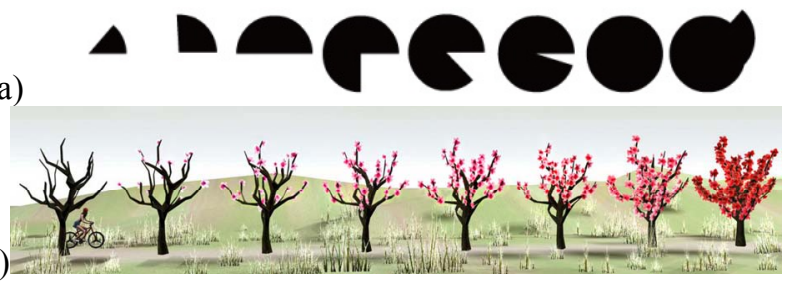

Fig. 4. a) shapes in gestalt theory; b) aesthetical tendency of a peach-tree. 


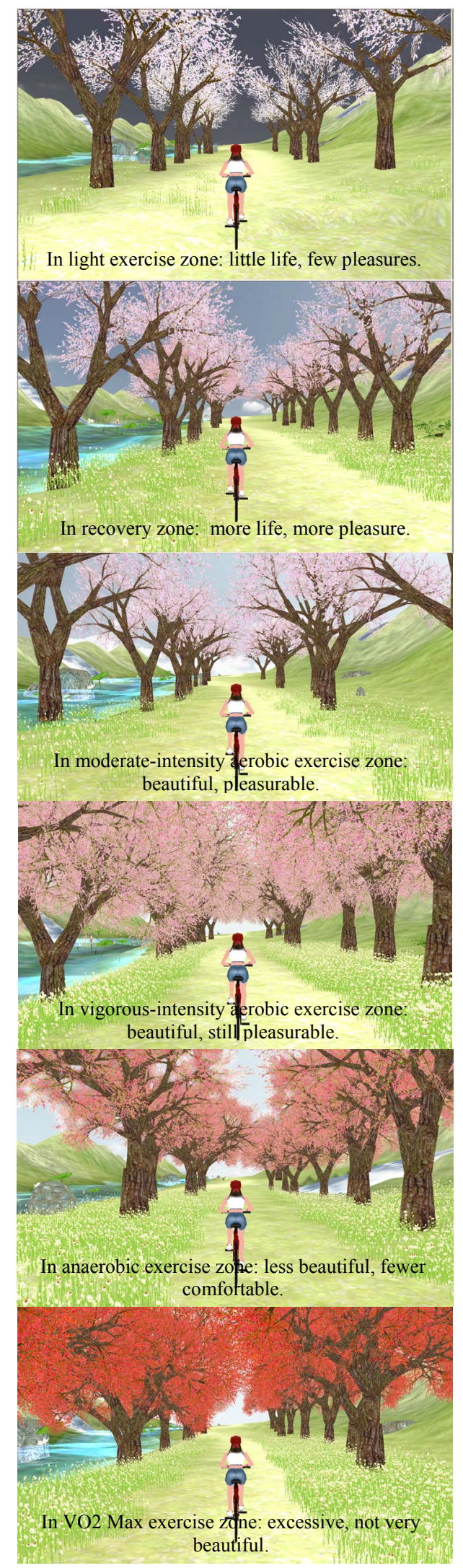

Fig. 5. Typical snapshots of our system. The adaptive interface is based on real-time biofeedback reflecting different training intensity via different audio-visual effects as well as varied aesthetic pleasure.

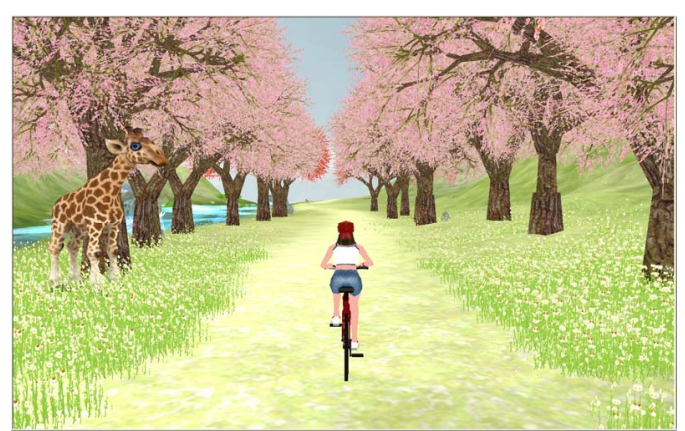

Fig. 6. An unexpected animal award could enhance the level of pleasure from novelty.

the newer and more awards she or he may get. By this way, users may feel aesthetical pleasure on traveling in a novel scene with full of curiosity.

As for a long term motivation, a large scale variation is necessary. We build an elements library to form different landscapes. The library consists of different kinds of terrain, texture, trees, awards, avatars, etc. Both of them can be selected and composed to a new scene before running the system. By this way, the system will always get a new life so that motivate the users to persist in.

\section{Reminder}

In the system, when an exercise session is finished, all the trees list horizontally in consequence and present like a heart rate visual trend line, as illustrated in Fig. 7. Users could intuitively find out the variation of their heart rate data and make evaluation of this time exercise intensity. Comparing to the traditional abstract heart rate trend line, this type of heart rate data visualization looks more beautiful and interesting. More importantly, in a long-term schedule, the accumulation of each time performance gives a helpful comparison of progress and frequency. This is a pleasurable reminder for users to do physical activity as scheduled.

\section{USER STUDY - VALIDATING PPM}

To evaluate the effectiveness and usability of PPM, we conduct a 3-week pilot in-lab experiment.

We invite 10 graduate students and 2 teachers (4 female and 8 male), aged from 20 to 45 with sedentary life style to take part in our test. The participants are divided into two groups, A and B, with 6 persons in each. Group A plays with our prototype system, while group B plays with a baseline system. The baseline system is a modified version in which all the relationships between player's physiological data and

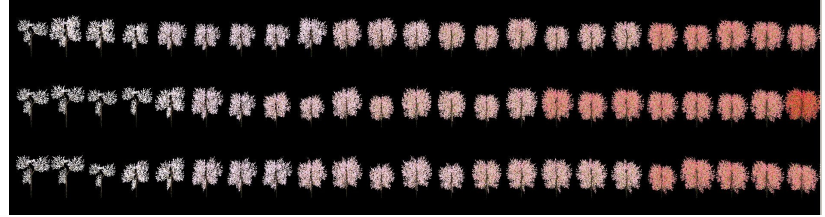

Fig. 7. The system ending picture is helpful on the comparison of progress and on the reminder of frequency (here is partly and in three times). 
scene's elements are being removed, but the real-time heart rate data in numerical value can be seen on the right corner of screen. The virtual scene of the comparison system has a random appearance of elements. This is an imitation of some kind of commercial system such as virtual roaming or cycling.

Each group was asked to exercise on two sets of equipment in two separate rooms, five times a week. During the test period, there is neither an indicator nor a spectator in the room, allowing participants to exercise freely as in their own home. Participants take turns to carry out their task. An exercise schedule is given for all the participants: 1) Warm-up (5 minutes of increasing intensity); 2) Cycling (20 minutes at $70 \%$ to $85 \%$ MHR(maximum heart rate )); and 3) Cool-down (5 minutes return to pre-exercise intensity). Although participants are advised that this program is good for their general health, they themselves need to decide the actual actions they take such as the intensity and duration of the exercise. What we are interested in is the duration and intensity control of their daily exercise sessions.

We gather all the heart rate recordings of each group and take them for the objective part of our evaluation. We then investigate how the heart rate signal behaved during the game play, by comparing the two systems. Fig. 8 shows the average heart rate trending lines of each group during the whole test period. In Fig. 8, the horizontal axis shows the elapsed time in minutes, while the vertical axis gives the heart rate level. Each trend line presents the heart rate reading of one participant during his whole exercise time on the fourth day.

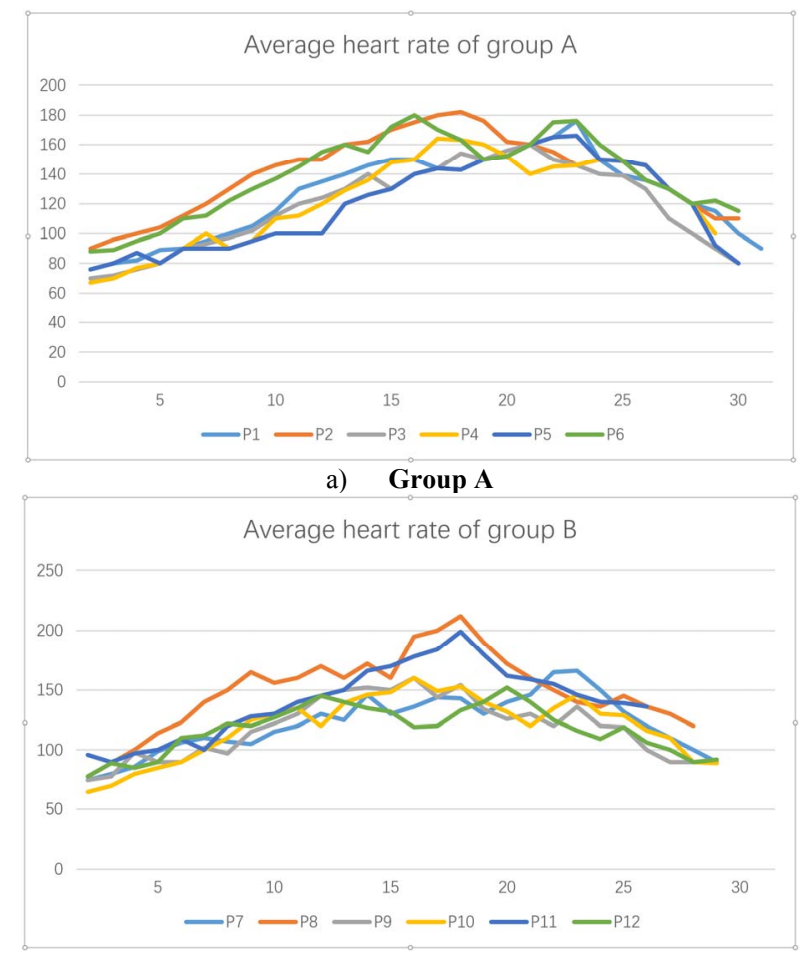

b) b) Group B

Fig. 8. The average heart rate trending lines of each group during the whole test period.
What we can obtain are not only the real time heart rate data recording of each participant, but also their actual exercise time in this case.

As it can be seen in Fig. 8 (a), the heart rate trend lines of all participants in group A were smooth and consistent. They all have a rising start and a relatively smooth afterwards in the middle, then a slowly decline to the end. In the middle section, almost all the trend lines stay in the area of $70-80 \%$ MHR. This tells that group A performs well on the three periods of the exercise schedule and its participants keep their heart rate in the target zone successfully. However, Fig. 8 (b) gives another picture: the trend lines look disordered and fluctuating. In the middle part, two curves linger at the zone of $60-70 \%$ MHR and two lines penetrate into the area of $80-$ $100 \%$ MHR. Only two line stays in the target zone. This may indicates that, although the numeric heart rate recording is given, participants in group B still hardly maintain their effort in the target zone. The insufficient duration time and the early ending clearly show that participants in group B are less motivated to finish the exercise as planned.

At the end of the test, each participant has been asked to complete a post-test questionnaire which consists of 5 questions as listed in Table 1. There are 5 questions in the post-test questionnaire, designed using the 7-point Likert scales $(1=$ 'strongly disagree' to $7=$ 'strongly agree'). Each participant gives his score from 1 to 7 . The mean ' $M$ ' and standard deviation, 'SD', are given below in Table 1.

From the table we can see that, on Q1, group A and Group $\mathrm{B}$ both got a higher mean score over 6 . This tells that aesthetic feedback and numerical feedback both work well on behavior guiding. In group $\mathrm{A}$, the visualization and incentive strategy has played their role significantly.

Q2 aims to investigate the important factor of exercise duration. Long enough duration indicates a good habit of doing exercise. Shorter duration time gives little exercise effect. The higher score of group A means that it is advantage of our system which encourages players to pursue sufficient exercise time and long term practice.

As to Q3, the group A again has a higher mean score which indicates that users are more likely to selects the system as their daily fitness exercise platform than a virtual roaming type. We can also see that participants in group A show high trust on the scientific aspect of the system.

In Q4, group B scores 3.0 while the group A give a highest agree in 6.9. This proves that PPM works well by interacting with users' personal real time physiological data.

Finally, we look at the answer to Q5, the difference of the mean score between two groups show a comparatively positive answer to group A. This means the system built on PPM shows its better quality both on physical exercise and aesthetical pleasure. The numerical feedback with ordinary static virtual roaming has a medium mean score which indicates a somehow boring experience. 
Table 1. Mean scores of two groups.

\begin{tabular}{|c|l|c|c|c|c|}
\hline & \multirow{2}{*}{$\begin{array}{l}\text { Are you agreeing with these } \\
\text { statements? }\end{array}$} & \multicolumn{2}{|c|}{ GroupA } & \multicolumn{2}{|c|}{ GroupB } \\
\cline { 3 - 6 } Q1 & $\begin{array}{l}\text { I can easily find my real-time } \\
\text { heart rate zone. I know how } \\
\text { and when to adjust or keep } \\
\text { the exercise intensity. }\end{array}$ & 6.8 & 0.6 & 6.2 & 0.5 \\
\hline Q2 & $\begin{array}{l}\text { I can easily maintain enough } \\
\text { exercise time every day and } \\
\text { really look forward to the } \\
\text { next time playing. }\end{array}$ & 6.5 & 2.2 & 3.5 & 2.6 \\
\hline Q3 & $\begin{array}{l}\text { I think the system is useful } \\
\text { for my scientific fitness } \\
\text { management. }\end{array}$ & 6.7 & 0.8 & 4.5 & 0.6 \\
\hline Q4 & $\begin{array}{l}\text { I believe the system fits my } \\
\text { fitness level well. }\end{array}$ & 6.9 & 0.3 & 3.0 & 0.6 \\
\hline Q5 & $\begin{array}{l}\text { The whole experience is } \\
\text { pleasure and wonderful. }\end{array}$ & 6.5 & 1.4 & 3.8 & 0.5 \\
\hline
\end{tabular}

\section{DISCUSSION}

Although a long-term study is needed to provide stronger evidence to support the design strategies of PPM, the results from the plot study confirm that at least a short-term trending is obviously positive.

Participants who used the prototype system give their comments on how they appreciated with the amazing guidance and awareness of their body state. They also explained that, because the physical intensity was mastered so easy and looked so interesting, they thought about their performance and commitment to being active more than usual. The participant $\mathrm{C}$ (female, age 27) expressed her interest of what her body state looked like:

I used to imagine the similarity between the life of trees, flowers and the life of myself. A numeric feedback cannot help on the recall of empathy. From the new system, I understand the energy of life clearly. I naturally know how to control my activity intensity. I love the association.

Most individuals are unlikely to perform the desired behavior for many reasons, especially afraid of the feeling of pain. The participant A (male, age 24) described what helps him to confront:

Regular activity is hard for me because exercise always makes me exhaust and tired... but in this kind of traveling, I forgot all the uncomfortable feelings. I even forgot the time. I just want to create a beautiful world of my own...

From the system evaluation, we find some extra learning of PPM. Personality is a factor we should consider more. Commonly a traveling environment is static, isolate and preset in a system. By utilize physiological data to adapt user interface, the virtual environment is no longer static or standard but personalized with close connection to the user himself. The user interface then becomes alive, sensible and valuable. At the same time, this kind of adaption has great identity to the user's current state, from body to mind. Both of these lead deep immersion of virtual traveling.
We also find that emotion plays a critical role in deep immersion. Similar to being intoxicated by the beauty of a painting, the pleasurable feeling of aesthetic natural beauty is helpful to merge oneself into the virtual environment.

\section{CONCLUSION}

The objective of this research is to give a possible solution of effective e-fitness system design. A pleasurable persuasive model was proposed with four design strategies including pleasure, guidance, encouragement, and reminder consist in. A prototype system was well developed based on this model. In the system, the physiological measures are coupled to the audiovisual aspects of a virtual world through aesthetic experience. The pleasurable emotion resulting from beautiful nature scenes, novel awards, and variable environmental elements encourage users to exercise both effectively and regularly.

There contributions lie in our study: 1) a designing model of pleasurably persuasive technology based on biofeedback to engage regular performance is proposed; 2) a prototype system is developed using the four design strategies and, 3) a between subject study on evaluating the PPM as well as system is carried out.

Our study on pleasurable persuasion technology can aid designers in creating exertion systems that users may feel engaging, increasing participation in physical activity and consequently allowing them to benefit from exertion. In the future we will improve PPM in theory, enrich the system presentation and, evaluate the model more thoroughly through long term user study.

\section{Acknowledgment}

This work is partially supported by NSFC (Grant No. 61332017), National project (Grant No. 2015BAK04B05), and Zhejiang Provincial Natural Science foundation of China (Grant No. LY15F020016). The research work of corresponding author, Zhigeng Pan, is co-supported by the Chang Jiang Distinguished Scholar Program. A great thanks to Qingru Meng, master of Wenzhou University, for his helpful work on the program implementation of the system. Many thanks to Huaqing Shen, associate professor of Zhejiang University, for his helpful advice concerning for the system design.

\section{References}

[1] Whitehead, A., Johnston, H., Nixon, N., \& Welch, J, "Exergame effectiveness: what the numbers can tell us," 5th ACM SIGGRAPH Symposium on Video Games, ACM, 2010, p. 5562.

[2] Haskell W L, Lee I M, Pate R R, et al., "Physical activity and public health: updated recommendation for adults from the American College of Sports Medicine and the American Heart Association," Circulation, 2007, 116(9): 1081-1093.

[3] Garriguet D, Janssen I, Craig C L, et al., "Physical activity of Canadian adults: accelerometer results from the 2007 to 2009 
Canadian Health Measures Survey,” Ottawa: Statistics Canada. 2011.

[4] Zhang M, Xu M, Han L, et al., "Virtual Network Marathon with immersion, scientificalness, competitiveness, adaptability and learning," Computers \& Graphics, 2012, 36(3): 185-192.

[5] Macvean A, Robertson J., "Understanding exergame users' physical activity, motivation and behavior over time," SIGCHI Conference on Human Factors in Computing Systems, ACM, 2013.

[6] Fogg B J., "Persuasive technology: using computers to change what we think and do," Ubiquity, 2002(December): 5.

[7] Locke E A, Latham G P., "Building a practically useful theory of goal setting and task motivation: A 35-year odyssey," American Psychologist, 2002, 57(9): 705.

[8] Prochaska J O., "Transtheoretical model of behavior change. Encyclopedia of Behavioral Medicine," Springer New York, 2013, p.1997-2000.

[9] Consolvo S, Klasnja P, McDonald D W, et al., "Goal-setting considerations for persuasive technologies that encourage physical activity," 4th International Conference on Persuasive Technology. ACM, 2009, p.8.

[10] Fogg B J., "A behavior model for persuasive design," 4th International Conference on Persuasive Technology, ACM, 2009, p.40.

[11] Baranowski T, Buday R, Thompson D I, et al., "Playing for real: video games and stories for health-related behavior change," American Journal of Preventive Medicine, 2008, 34(1): 74-82. e10.

[12] Berkovsky S, Coombe M, Freyne J, et al., "Physical activity motivating games: virtual rewards for real activity," SIGCHI Conference on Human Factors in Computing Systems, ACM, 2010, p. 243-252.

[13] Yim J, Graham T C., "Using games to increase exercise motivation," 2007 Conference on Future Play, ACM, 2007, p.166-173.

[14] Nacke L E, Kalyn M, Lough C, et al., "Biofeedback game design: using direct and indirect physiological control to enhance game interaction," SIGCHI Conference on Human Factors in Computing Systems, ACM, 2011, p.103-112.

[15] Boz I, Palaz S., "Factors influencing the motivation of Turkey's community volunteers," Nonprofit and Voluntary Sector Quarterly, 2007, 36(4): 643-661.
[16] Hamilton I, Imperatore G, Dunlop M D, et al., "Walk2Build: a GPS game for mobile exergaming with city visualization," 14th International Conference on Human-Computer Interaction with Mobile Devices and Services Companion, ACM, 2012, p.17-22.

[17] Lane N D, Lin M, Mohammod M, et al., "Bewell: Sensing sleep, physical activities and social interactions to promote wellbeing," Mobile Networks and Applications, 2014, 19(3): 345-359.

[18] Park T, Hwang I, Lee U, et al., "ExerLink: enabling pervasive social exergames with heterogeneous exercise devices," 10th International Conference on Mobile Systems, Applications, and Services, ACM, 2012, p.15-28.

[19] Buttussi F, Chittaro L, Ranon R, et al.,. "Adaptation of graphics and gameplay in fitness games by exploiting motion and physiological sensors," Smart Graphics. Springer Berlin Heidelberg, 2007, p.85-96.

[20] Lamoth C J C, Alingh R, Caljouw S R., "Exergaming for elderly: Effects of different types of game feedback on performance of a balance task," Stud Health Technol Inform, 2012, p.181: 103-107.

[21] Doyle J, Kelly D, Patterson M, et al., "The effects of visual feedback in therapeutic exergaming on motor task accuracy," Virtual Rehabilitation (ICVR), 2011 International Conference on. IEEE, 2011, p.1-5.

[22] Diakopoulos N, Kivran-Swaine F, Naaman M., "Playable data: characterizing the design space of game-y infographics," SIGCHI Conference on Human Factors in Computing Systems. ACM, 2011, p.1717-1726.

[23] Consolvo S, McDonald D W, Landay J A., "Theory-driven design strategies for technologies that support behavior change in everyday life," SIGCHI Conference on Human Factors in Computing Systems, ACM, 2009, p.405-414.

[24] http://ifholdings.com/Learn/Experience\#

[25] Barlow D, Durand V., "Abnormal psychology: An integrative approach," Cengage Learning, 2011.

[ 26 ]Nehamas A., "Only a promise of happiness," Princeton: Princeton UP, 2007.

[27] Arnheim R., "Visual thinking," Univ of California Press, 1969.

[28] Foster C, Florhaug J A, Franklin J, et al., "A new approach to monitoring exercise training," The Journal of Strength \& Conditioning Research, 2001, 15(1): 109-115.

[29] https://en.wikipedia.org/wiki/Gestalt_psychology 